\title{
CATWEB: A Tool for Developing Courses for the Web and from the Web
}

\author{
Luis Anido, Martín Llamas, Manuel Fernández and Juan Burguillo \\ Área de Ingeniería Telemática. Dpto. Tec. de las Comunicaciones \\ E.T.S.I. Telecomunicación. Universidade de Vigo \\ Campus Universitario 36200 Vigo. SPAIN \\ Tf : +34-86-812174, Fax: +34-86-812116 \\ e-mail : \{lanido,martin,manolo, jrial\}@ait.uvigo.es
}

Key words: Authoring tools, courseware, CBT, Internet, WWW

Abstract: $\quad$ CATWEB is a Course Authoring Tool for web-based educational environments. It provides a "one-piece" system to create and follow courses on the WWW. Its intended audience is teachers and instructors who are unfamiliar with computers and internet technologies. The many existing HTML editors and converters do not provide a suitable methodology for giving a pedagogical structure to developed documents. CATWEB provides this methodology by automatically structuring the pedagogical contents into learning blocks.

CATWEB instructors need only to know how to use a WWW browser; administrative and maintenance tasks are done by the system itself.

Furthermore, because CATWEB is based on standard internet services like FTP and HTTP, there is no need to set up additional software at the server or client side.

\section{INTRODUCTION}

During this decade we have witnessed the success of the World Wide Web as a medium to deliver educational material. Because it combines the advantages of distance education and computer based training capabilities

The original version of this chapter was revised: The copyright line was incorrect. This has been corrected. The Erratum to this chapter is available at DOI: 10.1007/978-0-387-35502-3_19 
(simulation, hypermedia material, etc.) the Web is a suitable platform where the learning process can be carried out successfully.

Unfortunately, most web-based teleteaching systems are related to scientific or technological subjects. The reason for this is the relationship between their authors and the world of new information technologies. Most of them are familiar with computers, the Internet and the Web. Therefore, it is very easy for them to develop new educational contents on the Web. However, this is not the case in most other subjects whose instructors are usually unfamiliar with these technologies. They could be given fantastic HTML editors and converters but these do not provide a suitable pedagogical relation between the materials and the documents developed.

Obviously, as teachers have changed their blackboards for overhead and slide projectors, changes in their teaching habits have been unavoidable; for example, the introduction of CBTs and WWW-based educational systems in their academic institutions.

As technically adept lecturers, we believe that we are responsible for providing a suitable set of tools to build educational environments for the Web. In this paper, we present a tool for developing such environments using the Web as a framework. Those who use our tool only need to connect their browser to a CATWEB server in order to build their courses from the WWW.

The next section reviews some currently available authoring tools on the Web. Then, section 3 outlines the main features of CATWEB, our approach to course authoring tools. Finally, some conclusions are presented.

\section{SOME AVAILABLE AUTHORING TOOLS}

Courseware is much more than several hypermedia documents linked to each other. Educational systems on the web need to provide a pedagogical environment where students feel as if they were in a virtual classroom, following the teaching methodology and assistance of their instructors. HTML editors and converters do not provide such an educational framework, and, therefore, several course-authoring tools have appeared in recent years. What follows is a summary of some of them.

\section{$2.1 \quad$ WebCT}

WebCT $[1,2]$ was developed at the University of British Columbia. It is targeted to create web-based courses through the Internet without any knowledge about the underlying technology. 
The WebCT server is based on a standard WWW server with some added functionalism to develop learning content. Designers of WebCT courses are allowed to use any of the following features:

- WebCT provides designers with the possibility of setting up the structure for a particular course as a linear or a tree structure. The students' traces are stored and the learning session can be resumed at any time.

- Glossary. Any word or concept can be included in the WebCT glossary with additional comments from instructors.

- External references in any page related to the actual learning content.

- Index Generation. Those words selected by the course designer will appear in the course index automatically.

- Questionnaires. Instructors are allowed to include multiple choice tests in the course. They are responsible for providing the correct answer as well as the amount of time the student will be given to complete the test.

The course designer is also responsible for including different communication tools in the course. Some tools available are e-mail, chat, and forums. Students may also be allowed to include private notes in the course pages.

Developers need only to know how to use a WWW browser. WebCT automatically creates every needed feature in a web-based course. Its value is accepted among the international scientific community.

\section{$2.2 \quad$ Learning Space}

Learning Space[3] was developed by Lotus. It is targeted for both academic institutions and industry. Apart from assisting instructors in the creation of educational content, it allows real-time lectures to be stored for subsequent use. The system is divided into different modules:

- The Media Center allows instructors to develop the course contents.

- The Assessment Manager allows instructors to monitor students' progress. It also allows editing of tests, questionnaires, and exams, which are delivered to the Scheduler to be included in the students' agendas.

- The Scheduler provides every needed facility to furnish students with a schedule so that they can know the objectives of every module, when they must be finished, and which questionnaires, tests, and exams must be completed.

- The Course Room permits the creation and management of different working groups.

- The Learning Space Central supports the general administrations of the course.

- The Data Bean Learning Service provides virtual lectures by teleconference, including real-time graphics, audio and video according to 
the ITU standard H.323; in this way it provides compatibility with tools like NetMeeting by Microsoft.

\section{$2.3 \quad$ Top Class}

Top Class [4] was created by WBT Systems. Its functionality can be easily classified according to the different Top Class modules:

- Top Class Creator simplifies course creation, editing of course content, and the, creation of questions of more than 20 different types.

Furthermore, this module permits presentation of different points of view about the same topic and different ways to explain the same concept. Thus, if the system detects that a student does not understand a given concept, it uses a different way to explain it instead of repeating the previous explanation.

- Top Class Converter converts Microsoft Office [5] documents, HTML[6] and RTF to Top Class format. Thus, external documents can be easily included in Top Class courses.

- Top Class Assistant allows Top Class documents to be edited with commercial tools by Microsoft like PowerPoint or Word. In this way, apart from using tools familiar for most instructors, TopClass makes it easy to integrate multimedia like video and animations using these commercial tools.

- Top Class Analyser is responsible for monitoring system use. From data generated by students' traces, it reports on the most used features and the efficiency of the learning process.

In addition, WBT provides a three-day course on how to use this system. The first day is intended to introduce the user to Top Class basic functionality and course structure. How to develop learning contents using available commercial tools, like those by Microsoft, is taught in the second day. Finally, on the last day, users learn concepts related to security and administrative tasks.

These systems are only a few examples of available web-based education authoring tools. From this brief survey, we want to emphasize the importance of importing external documents to new courses, as well as the creation of electronic quizzes and monitoring facilities. These features have been borne in mind and are included in our own authoring tool. 


\section{CATWEB: COURSE AUTHORING TOOL FOR THE WEB}

CATWEB is an integrated tool for developing and following courses on the web. It can be used by instructors and the system manager to develop their own courses, and by students to follow them. In this section, we first describe CATWEB as a tool for learning on the web and then we explain how to use CATWEB as a course-authoring tool. The section concludes with some technical remarks.

\subsection{Learning using CATWEB}

Using a Java-enabled browser, CATWEB students are allowed access to free-access courses and to those courses for which they are specifically authorised ${ }^{\mathrm{i}}$. Once a particular course is selected, its main page is displayed. This page provides links to other information pages about this particular course and the system, to the instructor's e-mail, and to every learning block in this course; see figure 1. Learning contents are organised into blocks to provide a suitable pedagogical structure for the course; related contents are always in the same learning block. Instructors are allowed to impose a particular sequence on this learning block structure; e.g., the first learning block must be completed before accessing the second one, etc.

Different navigational structures can be set up and the course designer can thus adjust the current performance of the CATWEB tool to different pedagogical methodologies. CATWEB simply tries to reflect the way teachers are used to teaching and the way students are used to learning. 


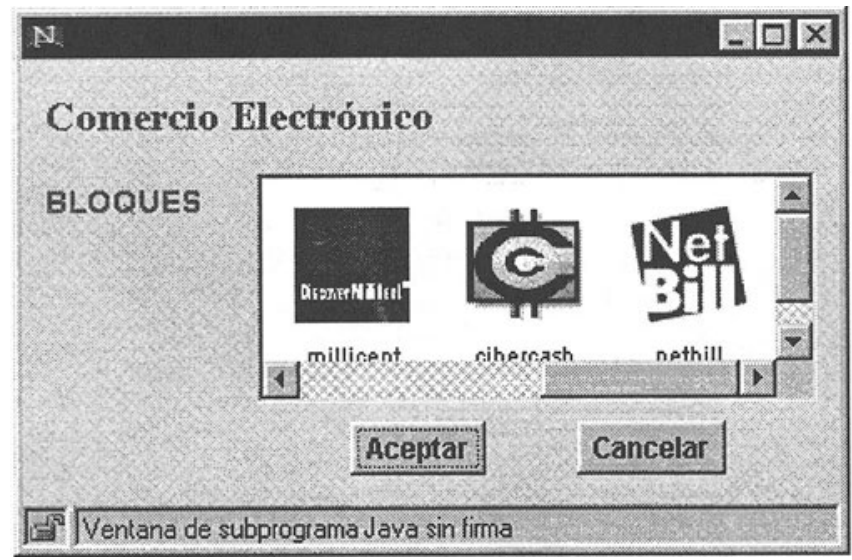

Figure 1. Learning blocks in a course about electronic commerce

Every learning block includes the following parts:

- Preferences to be set by the student. Page formats, foreground and background colours, etc., for this particular learning block.

- Short notes about the learning block. Their aim is to clarify aspects of its contents, which learning blocks should be accessed before and after this particular one, and so on.

- Links to other related sites in the WWW. CATWEB allows students to come back to the previous point in the learning block after "surfing" the web.

- Private notes. Such notes, posted by a student, are accessible only by the poster.

- News. This tool allows students and teachers to share information, comments, notes, etc., about the learning contents of the block.

- Chat. Several people (students and teachers) can be involved in real-time communication. Every chat room is identified by the particular subject of the conversation. Thus, any learner or instructor can choose the room most appropriate to her or his interests at a given time.

- Lessons. These include whatever multimedia materials have been provided by their developers; see figure 2 . Of course, they are the core of every learning block.

- Quizzes. These allow students to check their progress in the learning process. Every quiz is shown to students as the instructor who developed the course defined it. 


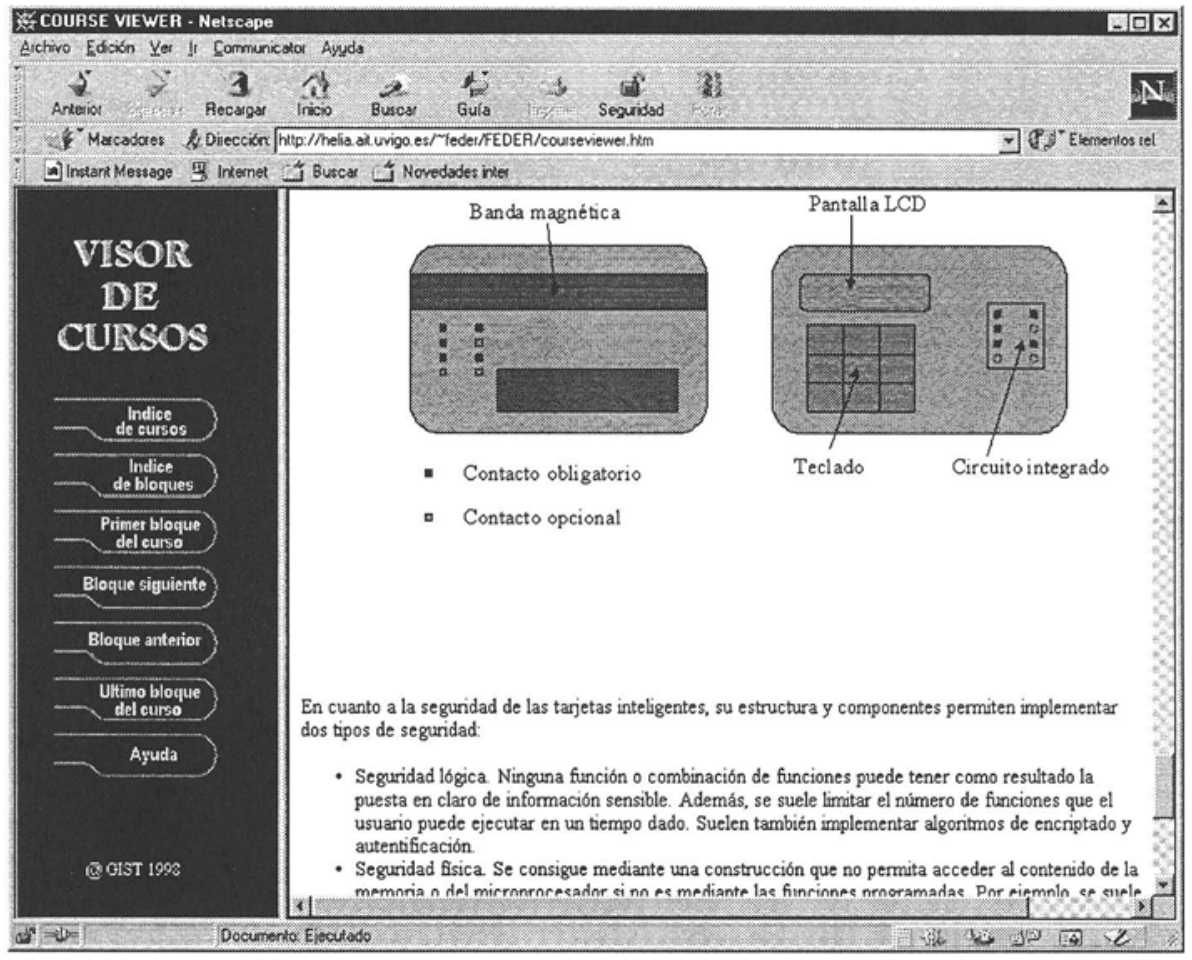

Figure 2. Learning using CATWEB

Figure 2 shows the CATWEB course viewer. The left frame provides any feature necessary to navigate across the course: course index; learning block index for this particular course; links to the first, previous, next, and last learning block in this course. In addition, there is context sensitive help that can be accessed at any time and that explains what the student is allowed to do according what has been done before. The right frame shows any multimedia material included in the course lessons.

\subsection{Developing learning contents using CATWEB}

The system manager and those instructors defined by the system manager are allowed to develop their own CATWEB courses. They need only to know how to use a WWW browser because CATWEB allows teachers to develop their web-based courses from the WWW. There is no need for CATWEB developers to set up additional software on their computers, 
thereby avoiding what can be a daunting task for those unfamiliar with such things.

First of all, CATWEB provides an easy to use management application to add new students to a particular course and maintain up-to-date information about them. Teachers can add new students one by one, see figure 3 , or they can upload a file stored in their computers containing the new students' data. Of course, instructors are allowed to delete, update, or check information about any student of any course for which they are responsible.

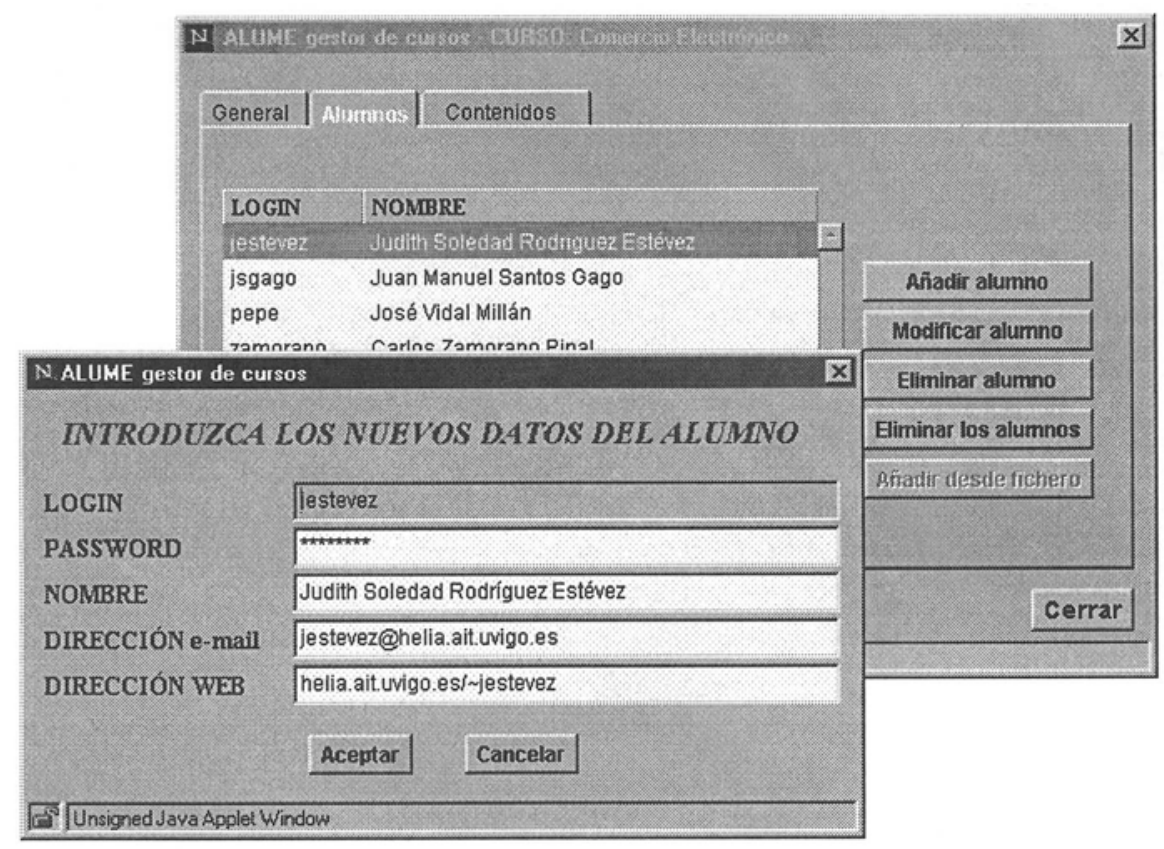

Figure 3. Administrative tasks in CATWEB

As CATWEB learning contents are organised in blocks, instructors are allowed to add, modify, and delete new learning blocks in their courses. There is no need to use or install other applications. CATWEB course designers need only to connect to the system, which makes the rest of the structure for them.

Whenever CATWEB course developers create or modify a particular learning block they can set up the following features:

- Learning block name and some default format parameters: page format, foreground and background colours, etc. Every student features to suit personal preferences can change these. 
- Instructors may decide that this particular block can only be accessed by those students who complete other learning blocks successfully ii. CATWEB permits assisted or free navigation according to the particular learning content of every course and the instructor's discretion.

- Short notes and related links to be included in this learning block.

- The instructor's private notes for this particular learning block.

- Topics that should be discussed at the news forum by students. These predefined topics should be those which usually would be discussed by trainees learning this particular subject.

- The subject of the different virtual communication rooms. Thus, instructors can set up default topics to be discussed by learners.

- The learning contents itself: the lessons. For every new page in the lesson, they can set up their format and contents and can upload any new multimedia material they want from their own computers or any other host at the Internet. They are also allowed to import data in Microsoft Office format, HTML, and RTF for their courses. Figure 4 shows how instructors are allowed to edit the pages of any lesson. Editing, as well as the other CATWEB features, is done as shown in figure 4, using a standard web browser, thereby avoiding the need for additional software.

- Quizzes. A broad range of options is available for every quiz included in the learning block: number and titles of questions, type (short answer, multiple choice, checkbox, etc.), how many times students can access the same question, whether or not to show correct answers to students, time to complete the question, whether or not to send students' answers to their instructors by e-mail, generation of statistical data, and so on.

The instructor responsible for a particular course can follow students' progress in the course. Students' traces are stored on the server and are accessible to instructors.

In addition, CATWEB provides a useful feature to transform the webbased course into a stand-alone CD-ROM format. Thus, students can follow the same courses with the same structure but in a stand-alone way without any Internet connection.

The CD-ROM version avoids the network overhead that may interfere with the students' focus on course content. On the other hand, the Internet version always provides the most recent version both of course content and of the CATWEB tool itself. This trade-off must be borne in mind in making the proper choice to meet particular needs. 


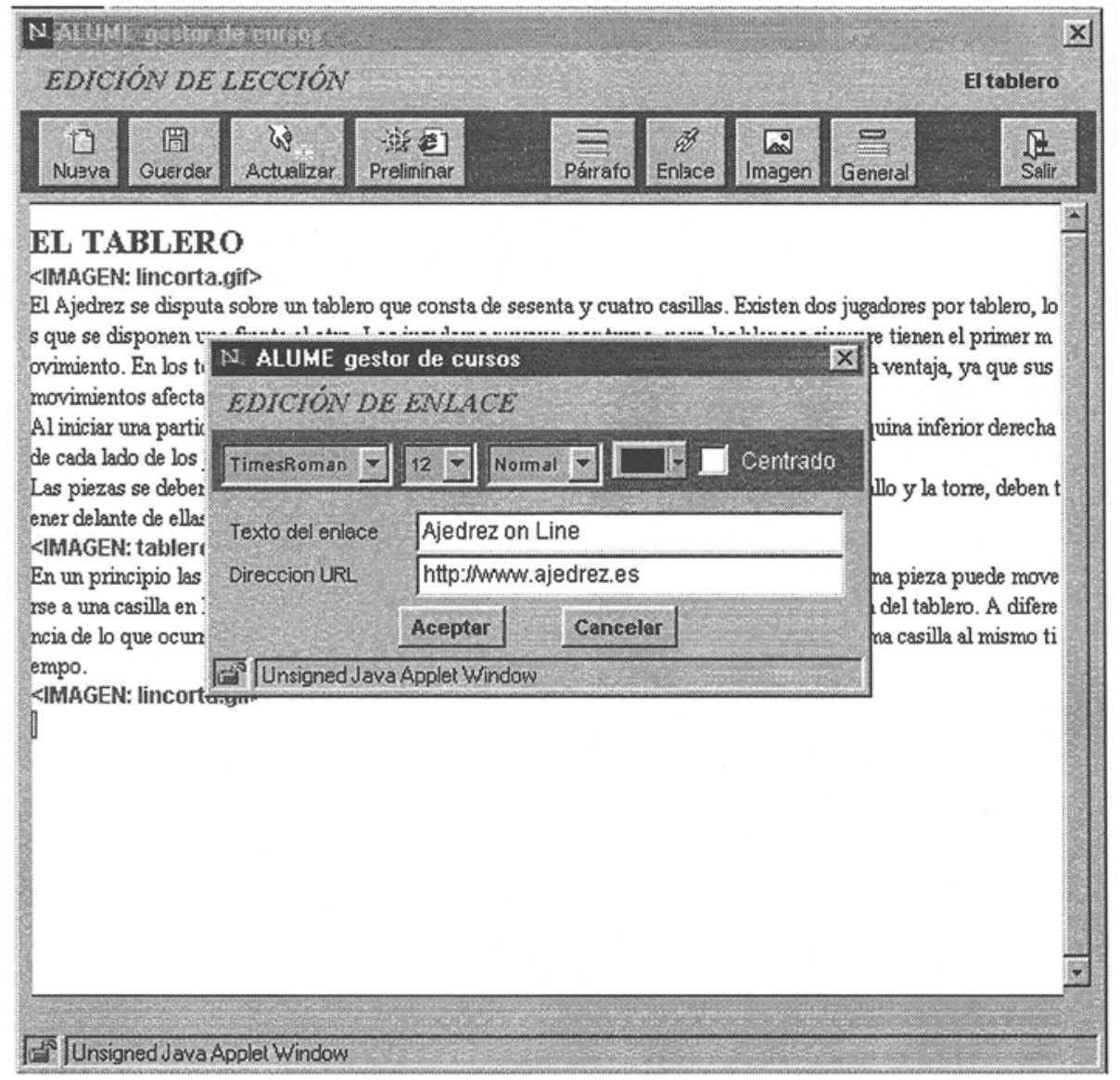

Figure 4. Creating learning contents using CATWEB

\subsection{Brief Technical Remarks about CATWEB: A Server-less Architecture}

Because we wanted CATWEB to be as easy to use as possible, we have aimed at an easily to set up and an easily maintained architecture. The CATWEB course viewer and authoring tool need only some standard internet services. There is no need for additional software at the client side or additional server applications at the server side.

As shown in figure 5, our system is based in two standard internet protocols: HTTP protocol [7] and the FTP protocol [8]. A standard web 
server sends any multimedia material to the client's side. These contents are managed by Java applets at the clients' computers, which need only a Javaenabled browser. Additional files to provide any CATWEB functionality are upload and download using the File Transfer Protocol.

The main Java applet at the client's side connects to the FTP port at the server computer and, following the standards of the FTP protocol, manages any needed data at the server side.

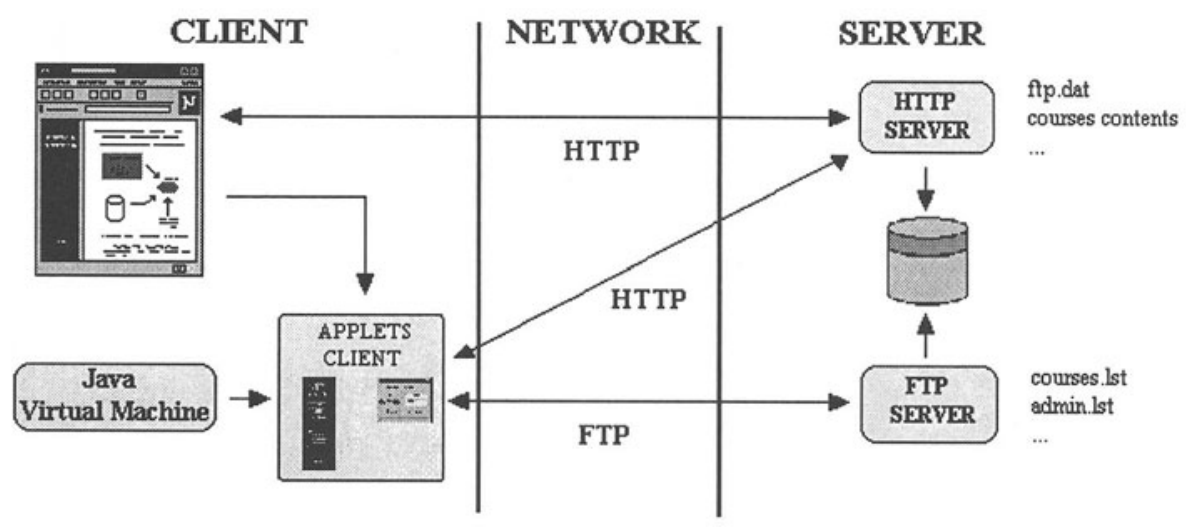

Figure 5. CATWEB Architecture

We found that the Java applets raised a security exception when the FTP server tried to connect to the client side. Java applets do not allow external connections to the machine on which the browser is running. To solve this problem and to avoid external connections to the client computer, we decided to use the ftp standard service to exchange data between two ftp servers, one active server and one passive server. Since every connection is established from the active side to the passive side and the data exchange can be controlled by a ftp client, we found this solution suitable for our system. Here, the CATWEB applet is both the ftp client and the ftp active server. Therefore, every TCP/IP connection comes from the client side to the server side, and all necessary data can be stored there without any particular CATWEB server.

We need only standard HTTP and FTP services at the server side. The main functionality has been taken to the client side following the real distributed architecture paradigm. In fact, the HTTP server sends only course pages (HTML documents). The FTP server's main job is to store students' current state information and instructors' newly developed courses.. 
Thus, CATWEB has taken full advantage of existing internet services to provide every CATWEB feature. Because there is no need for a particular CATWEB server, there is no special server maintenance. At this point, we should remember the main philosophy of CATWEB: to allow teachers unfamiliar with the Internet to provide their own web-based courses.

\section{CONCLUSION}

Our tool's aim is to ease the task of those developers of web-based courses who are not familiar with internet-related technologies. As far as we know, there are many HTML editors and converters, but very few of them provide a pedagogically suitable way to relate new contents.

CATWEB is able to provide an environment where the creation of new educational material for the Web is easy enough for those who are unfamiliar with the Internet and computers. The corresponding educational framework is automatically provided by our system. Instructors need only concern themselves with what they want to teach to their students.

In addition, CATWEB provides a course viewer to follow these courses using any conventional internet browser. Thus, the CATWEB tool allows teachers to develop courses and students to follow these courses using a "one piece" environment. Furthermore, because CATWEB needs no additional software at the server side, no specific CATWEB server is needed, thereby eliminating special set up and maintenance tasks.

The CATWEB authoring tool is now being used to develop and follow several courses at our university. We are concerned about the importance of course authoring tools for those who are unfamiliar with computers and the Internet. We are constantly collecting requirements from our "non-technical" colleagues in order to improve our tool. Instructors need to concentrate on educational content, not on how to implement them technically; that is the final aim of CATWEB.

\section{ACKNOWLEDGEMENTS}

We want to thank the European Union and the Spanish government for their partial support for developing CATWEB under projects FEDER "PTF: Plataforma de TeleFormación" (FEDER 1FD97-0100) and "Diseño y Desarrollo de Software Documental sobre Internet e Intranet" (FEDER 
1FD97-0282). We also want to thank Mr. David Agra, Mr. Juan Manuel Santos and Mr. Carlos Zamorano for their contribution to the development and testing of CATWEB.

The first author particularly wants to dedicate this work to Mr. Juan A. Aguado Vázquez.

Finally, we want to thank those lecturers from the Telecommunications Engineering School at the University of Vigo who have used CATWEB to create new learning content for the Web.

\section{REFERENCES}

[1] M.W. Goldberg and S. Salari. An update on WebCT (World-Wide-Web Course Tools)- a Tool for the Creation of Sophisticated Web-based Learning Environment. Proceedings of NAUWeb'97- Current Practices in Web-based Course Development, June 12-15, 1997, Flagstaff, Arizona, USA.

[2] WebCT homepage: http://homebrew1.cs.ubc.ca/webct

[3] Learning Space homepage: http://www.lotus.com/products/learningspace.nsf/

[4] Top Class homepage: http://www.wbtsystems.com/soluctions/products.html

[5] Microsoft Office homepage: http://www.microsoft.com

[6] HyperText Markup Language: http://www.w3.org/MarkUp

[7] HyperText Transfer Protocol: http://www.w3.org/Protocols

[8] File Transfer Protocol: http://www.army.mil/ftptutor.htm

\footnotetext{
${ }^{i}$ Developers of new CATWEB courses are allowed to mark them as free-access (everyone can use them) or restricted-access (students must be included in those courses to gain access to them). A course may require completion of other courses for access. Thus, instructors can decide that their students have to complete one/several course/s before accessing another one.

ii "Successfully" here means to access every lesson and to get some predefined marks on the quizzes.
} 